\title{
THE DYNAMICS OF GLOBULAR CLUSTERS IN HIGH ECCENTRICITY ORBITS
}

\author{
R. K. Bhatia \\ Royal Observatory, Edinburgh
}

The aim of the present work is two-fold: 1) To study the effect of the tidal field of the Galactic bulge on a cluster in a high eccentricity plunging orbit with half-mass density nearly equal to the Roche density; 2) To estimate the tidal or limiting radius for such orbits.

Present evidence seems to support the idea that globular clusters move in high eccentricity orbits (e.g. Castellani and Melchiorri 1981; Caputo and Castellani 1984), although Innanen et al (1983) have argued for moderate eccentricity orbits. Tidal effects become very strong for clusters in high eccentricity orbits with small pericenter distances. For such an orbit, after pericenter passage, a cluster will lose appreciable mass and expand.

Representing the cluster by a 500-particle system, we have performed N-Body simulations using the code TIDAL kindly made available by Sverre Aarseth. The collision parameters are:

Ratio of cluster mass to mass of galactic bulge: $10^{-5}$

Eccentricity of orbits studied: $0.6,0.65,0.68,0.70,0.8,0.9$.

Pericentre distance: Chosen such that the half-mass radius equals the tidal radius computed from King's formula $(<1 \mathrm{Kpc})$.

Fig. 1 displays the variation with eccentricity for a) $\Delta U /|U|$, the fractional change in the total energy of the system, b) $\Delta U_{B} /|U|$, the fractional change in energy of the bound system, c) $\Delta M / M$, the fractional change in mass, and $d$ ) the fractional change in half-mass density normalized to the density for $e=0.9$

Note the sharp increase near $e=0.7$; also the increase in $\Delta M / M$ falls below that for $\Delta \mathrm{U}_{\mathrm{B}} /|\mathrm{U}|$, implying that the gain in energy is going more towards the expansion of the cluster rather than for expelling mass. Indeed, for $e=0.6$ the cluster disrupts completely. 

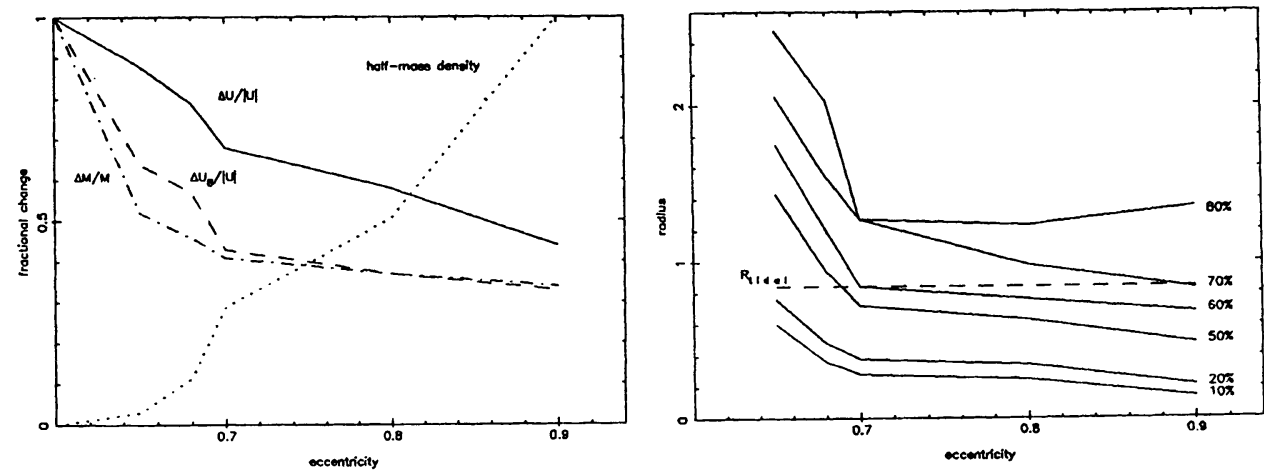

Fig.1. $\Delta U / \mid U_{\mid}$etc. vs. e

Fig. 2 Radius containing $10 \%$ mass etc. vs. e

Fig. 2 displays the radius containing $10,20,50,70$ and 80 per cent masses as a function of eccentricity. The dashed line indicates the theoretical tidal radius. The expansion increases with decreasing eccentricity: for $e=0.68$, the half-mass radius increases by a factor of 1.4 and the radius containing $80 \%$ mass by a factor of almost 3. Indeed, as the dotted line shows in Fig. 1, the half-mass density for $\mathrm{e}=0.68$ is nearly 9 times less than for $e=0.9$. It is well known that halo globular clusters in the Galaxy have very low densities. Is it possible that some of them have had a close encounter with the Galaxy which has caused them to expand?

An idea of the tidal radius of the clusters in high eccentricity orbits can be obtained from Fig. 2. In all the cases, the final radius of the cluster is much larger than the theoretical tidal radius by a factor of up to 3 . We therefore conclude that for halo clusters, the tidal radius computed from King's formula can be too small, depending on the eccentricity of the orbit and on the pericenter distance.

To summarise: 1) The final structure of a cluster after collision depends critically on the collision parameters and on the eccentricity of the orbit. If halo clusters move in orbits of high eccentricity, they can undergo considerable change in their structure after pericenter passage, leading to a considerable expansion of the cluster. 2) For such orbits, the tidal radius computed from King's formula is much smaller than that suggested by simulations.

\section{REFERENCES}

Caputo, F. and Castellani, V. 1984 Monthly Notices Roy. Astron. Soc. 207, 185.

Castellani, V. and Melchiorri, M. 1981 Astrophys and Space Sci. $89,289$.

Innanen, K. A., Harris, W. E. and Webbink, R. F. 1983 Astron. J. 88,338 . 